\title{
The method of local impact for the analysis of structures for progressive collapse
}

\author{
Sergey Saiyan ${ }^{1, *}$, and Alexander Paushkin ${ }^{1}$ \\ ${ }^{1}$ Moscow State University of Civil Engineering, Yaroslavskoe shosse, 26, Moscow, 129337, Russia
}

\begin{abstract}
A method for calculation on the progressive collapse, in accordance with which the account of the fall of ceiling panels on the underlying floor when the loss of bearing capacity of vertical frame elements in the form of local impact determined by multiplying the weight plates on the dynamic coefficient determined by the drop height and static deflection. The calculation is carried out in several stages with the use of the LIRA SAPR software.
\end{abstract}

\section{Introduction}

The calculation of progressive collapse is performed in connection with the need to ensure the reliability of the structure for a long time of its operation and ensure the survivability of the building in emergency situations arising from the action of special loads and impacts on the stages of construction and operation of facilities, as well as during reconstruction and overhaul [1].

Emergency situations include special loads and impacts that occur as a result of:

- material defects;

- poor quality of work;

- design errors, including those caused by imperfection of normative documents for design;

- violations of operation of buildings, including their industrial or engineering equipment;

- violations of technological process, temporary malfunction or breakage of the equipment and for other, unknown reasons [2].

When designing structures, scenarios for the implementation of the most dangerous emergency situations should be developed and strategies developed to prevent the progressive collapse of the structure in the local destruction of structures. A progressive (in the form of an avalanche) collapse is understood as the spread of initial local damage in the form of a chain reaction from one element to another, which ultimately leads to the collapse of the entire building or disproportionately large part of it [2].

*Corresponding author: berformert@gmail.com 


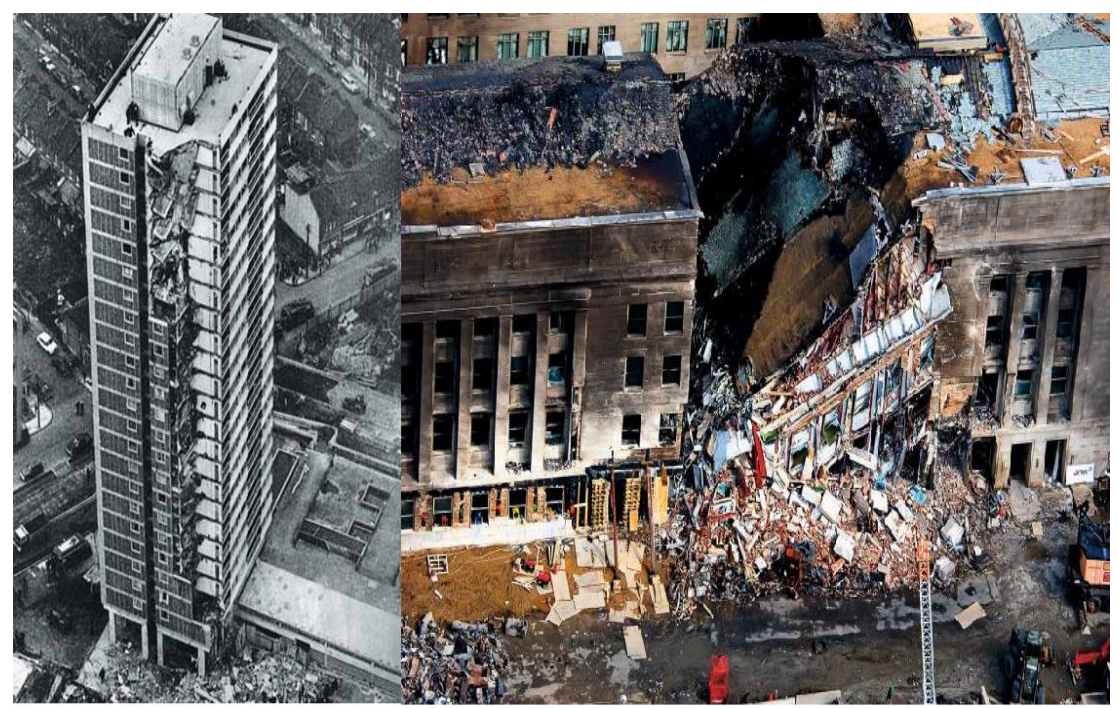

Fig. 1. Progressive collapse of buildings

As can be seen from (figure 1) the initial local damage to the structural element led to a chain reaction of destruction in the vertical direction but did not destroy the building as a whole.

At the moment there are several methods to perform the calculation of the progressive collapse. One of the known is the kinematic calculation method using the theory of limit equilibrium [3]. Its essence is to determine the operation of internal forces and external loads on the possible movements of the mechanism in question.

In this paper, we propose a method of local impact and the results of the calculation of the conditional building by this method. The impact caused by the fall of the slab causes a dynamic impact, and as a result, a sharp jump in stresses and strains in the local area of the underlying structures. In determining the dynamic loads, an engineering approach is used from the standpoint of the approximate theory of impact, when the dynamic deflections and stresses are determined by multiplying the static values by the dynamic coefficient depending on the height of the fall and static displacement:

$$
P=P_{s} \cdot \mu
$$

where: $\mu=1+\sqrt{1+\frac{2 h}{\lambda_{s}}}, h-$ the drop height of the body, $\lambda_{s}-$ static deflection.

Strength and deformation characteristics of materials are accepted in accordance with the applicable regulations [1]. The calculation was performed on a combination of loads consisting of constant, long and one of the special, taking into account the coefficients of the combinations. 


\section{Research}

For the purposes of testing method is considered reinforced concrete building with overall dimensions $60 \times 24 \times 15 \mathrm{~m}$, with monolithic slabs and columns (grid columns 10x6m) loaded by permanent and long loads (figure 2 ).

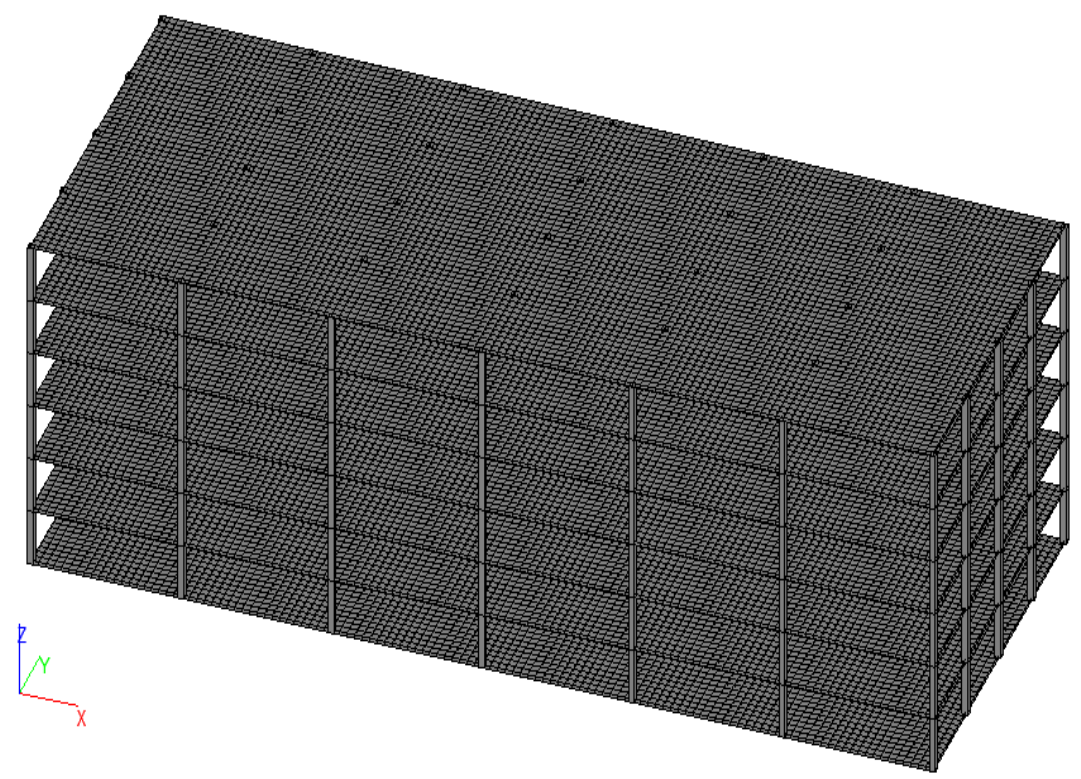

Fig. 2. Scheme monolithic framework

The method is based on the following sequence of events. It is assumed that under the most loaded floor, one of the most stressed columns loses its bearing capacity and is removed from the design scheme, and, if the upper plate is unable to perceive the effort, it falls on the underlying plate.

The choice of the most loaded floor is determined by the results of a preliminary linear calculation of the entire building for static loads. Select the floor in which the elements have the greatest effort and stress. The failure of such elements is the worst way to affect the work of the whole structure, as the design seeks to redistribute large efforts to neighboring elements.

The value of the static deflection of the underlying overlap required to determine the dynamic coefficient is calculated in advance from the static calculation of the entire building. In the next stage, the whole building is calculated for the action of the local impact load applied to the underlying slab. If the calculation shows the loss of bearing capacity of the underlying elements, it continues for the underlying floors.

In our example, the fourth floor is the most loaded. The most dangerous scheme of collapse was chosen out of the work of one of the far-right columns of the third floor under the action of the greatest bending moment $M_{y}$ acting along the larger building (figure 3 ). 


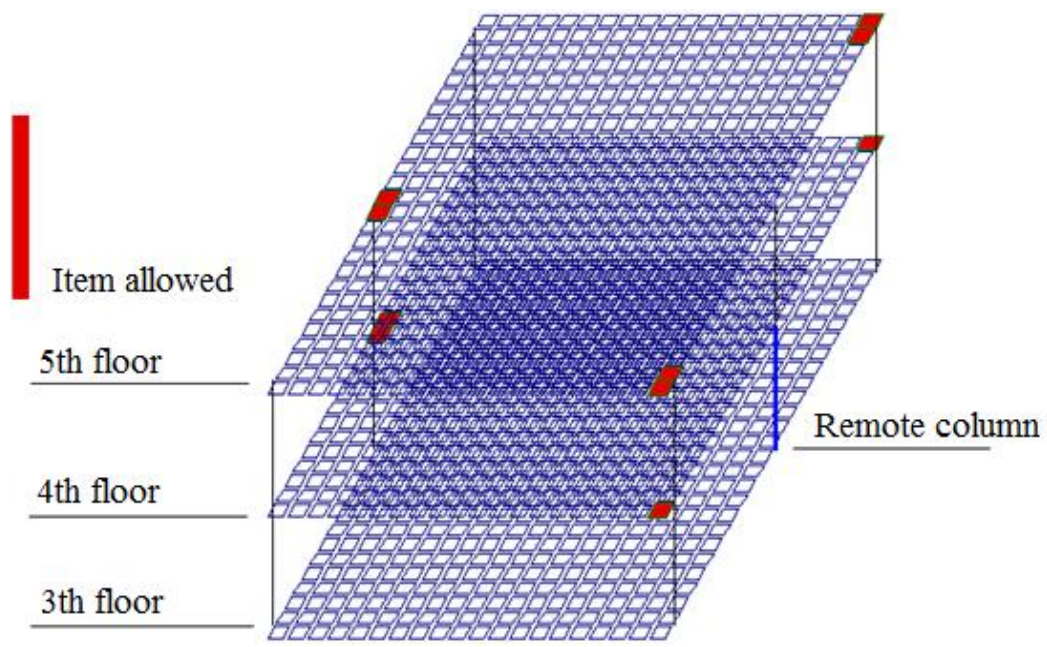

Fig. 3. A fragment of the local calculation area with the location of the removed column

In order to identify the most dangerous version of the collapse was considered the exit from the work of one of the central columns of the third floor. The calculation showed that despite the heavy load on the central columns, the collapse is more extensive in the extreme spans of the building. Due to the extreme location of the columns and their rigid interface with the floor slab, in the places of connection of columns and plates there are large bending moments, which determines the loss of bearing capacity of structures, which is mainly associated with the development of tensile stresses.

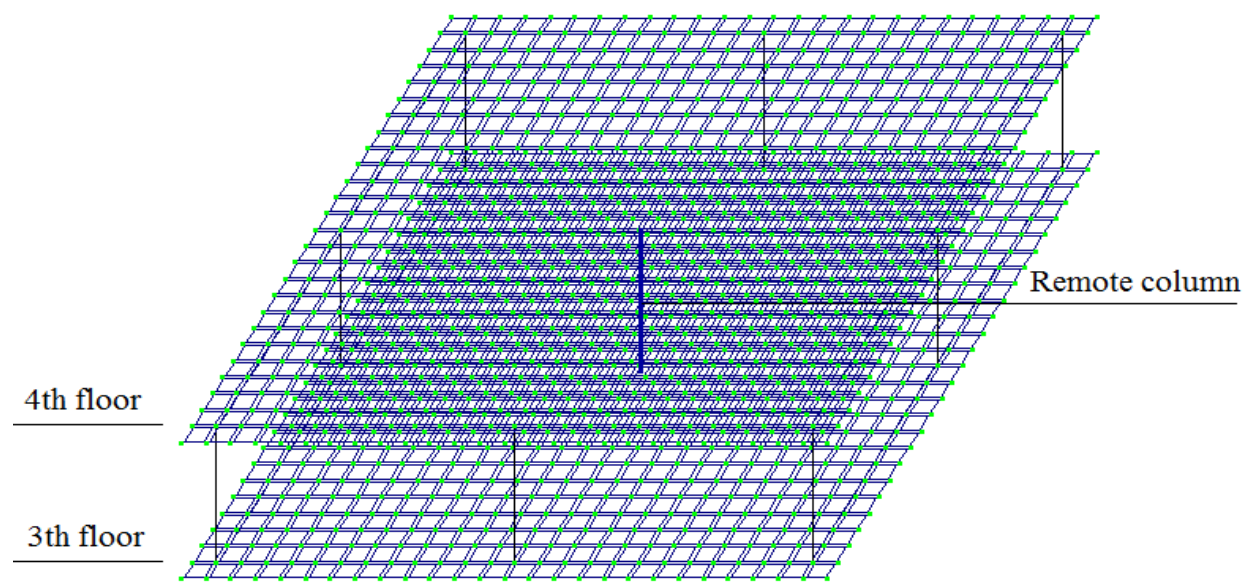

Fig. 4. A fragment of the area with the location of the retracted column of the central span

Set the special (emergency) impact, removing the extreme right column of the third floor of the design scheme.

Based on the nonlinear calculation we have the destruction of elements near the columns in the considered area of collapse of the fifth and fourth floors (darkened areas). 
Adding to this analysis results of the depth and width of fracturing, we have a significant probability of a progressive collapse along the contour of the plate.

Since the collapse in equal probability is subject to 2 floors, it is possible to consider 3 mechanisms of their collapse:

A) The covering of the fourth floor is in the boundary state close to the loss of bearing capacity (further slight increase in the load will lead to its collapse), the ceiling of the fifth floor falls to the fourth floor, causing an inelastic blow, which will further lead to the fall of the plates of the fifth and fourth floors to the third floor.

B) The ceiling of the fifth floor is in the boundary condition, the ceiling of the fourth floor collapses on the third floor.

C) The ceiling of the fifth and fourth floors collapse at the same time. Due to the different height of the fall on the third floor, the fourth floor collapses first.

The boundary state of the floor slabs of the fourth and fifth floors is possible.

This case was not considered because of a rather small probability.

In this paper, case B was chosen as the easiest to calculate. The scheme of distribution of destruction in the considered area is shown in figure 5.

Based on the results of the calculation, we have a local effect of destruction on the overlap of the third floor from the fall of the fourth-floor plate.

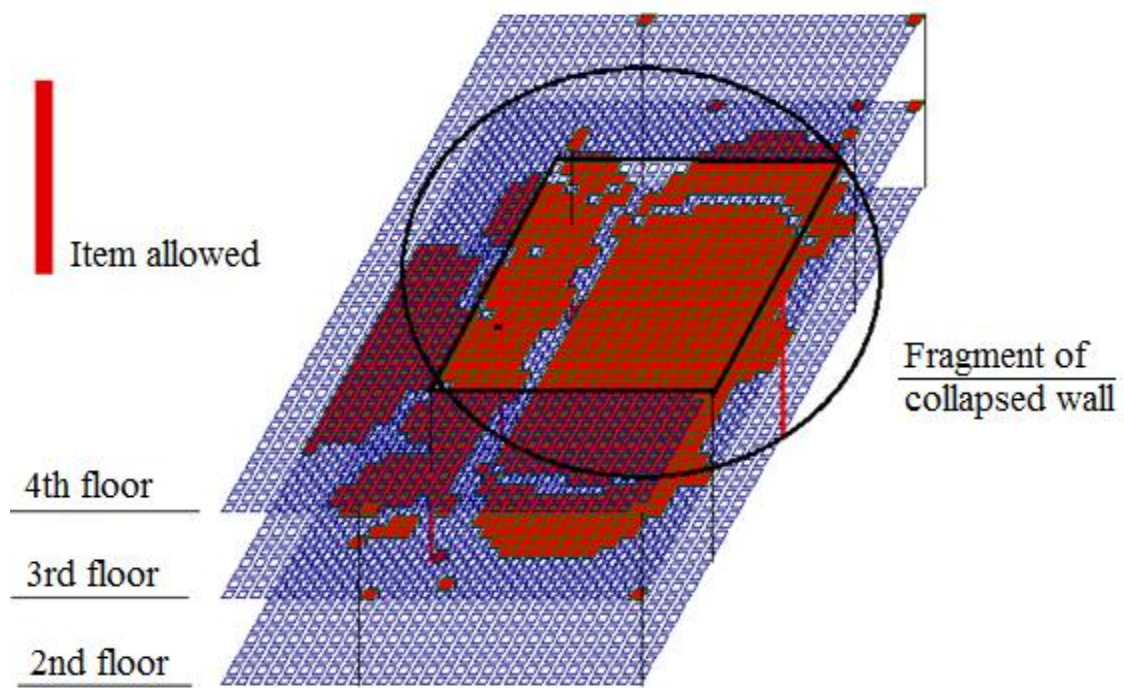

Fig. 5. Propagation of destruction in the considered area

The effect of destruction spread by chain reaction further along the scheme, destroying the columns of the 2 nd and 1st floors, and damaging the elements of floor slabs located near the local collapse (figure 6).

The damage is more distributed in the vertical direction, which in turn forms areas of high stress on the horizontal elements near the destroyed columns.

The building is subject to progressive collapse and a chain reaction of falling plates in the area under consideration will lead to a complete collapse of this area, but will not bring down the entire building as a whole.

Due to the rigid coupling of the frame elements together, the structure is able to redistribute forces from the destroyed elements to neighboring ones, which leads to the damping of the effects of dynamic impact from the fall of the slab at a distance from the 
local area of collapse. This factor helps to reduce the impact of dynamic effect on the building.

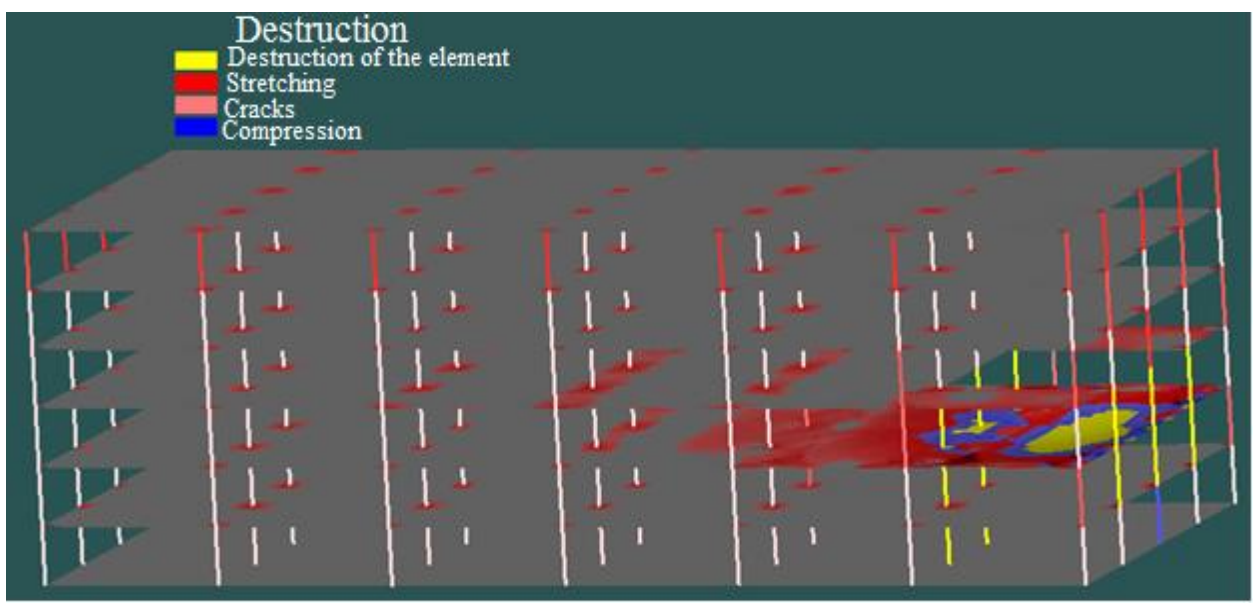

Fig. 6. Building destruction scheme

\section{Conclusions}

1. For the first time the method of calculation on a progressive collapse with application of the concept "local impact" is offered.

2. Trial calculations have shown the applicability of this method as one of the options for calculating structures for progressive collapse.

3. The calculation for a progressive collapse is probabilistic and requires taking into account all possible scenarios of destruction.

4. Step-by-step of the calculations made allows you to control more of the input parameters in the calculation than a single simulation of buildings and structures on the progressive collapse in the time interval.

5. Progressive collapse is more extensive in the outermost spans of buildings and structures.

\section{References}

1. GOST 27751-2014 Reliability of building structures and bases. Fundamentals

2. SP 296.1325800.2017 Buildings and structures. Special effects

3. G. I. Shapiro, J. A., Eisman, J. M. Strugatsky, Recommendations for the prevention of progressive collapse of large-panel buildings (1999)

4. G. S. Vardanyan, V. I. Andreev, N. M. Atarov, A. A. Gorshkov, Resistance of materials with the basics of the theory of elasticity and plasticity, 638 (2011) 